\title{
Time efficient method for defocus error compensation in tomographic phase microscopy
}

\author{
J. Kostencka, T. Kozacki, M. Dudek and M. Kujawińska \\ Institute of Micromechanics and Photonics, Warsaw University of Technology, Św. A. Boboli 8, 02-525 Warszawa
}

Received July 01, 2014; accepted September 22, 2014; published September 30, 2014

\begin{abstract}
We present a holographic method for defocus error compensation in tomographic phase microscopy, which enables high quality reconstruction in the presence of a meaningful run-out error of the measurement system. The proposed method involves indirect determination of the sample displacement from the in-focus plane. The sought quantity is deduced from the transverse movement of the rotating sample, which can be determined with high precision using correlationbased techniques. The proposed solution features improved accuracy and reduced computation time compared to the conventional autofocusing-based approach. The validity of the concept is experimentally demonstrated by tomographic reconstruction of an optical microtip.
\end{abstract}

Tomographic phase microscopy (TPM) is a laser interferometric method enabling quantitative measurement of micro-scale semi-transparent samples. Its main advantage over conventional interferometric techniques is the possibility of charactering a three-dimensional (3D) internal structure of the specimen, instead of providing integrated information about phase delays [1]. In TPM, a laser interferometric (or digital holographic) microscope is used to obtain a set of quantitative phase images of the sample collected at various illumination directions in the range $0-180^{\circ}$. During the measurement, the scanning of a relative angle of illumination with respect to the sample is usually achieved by rotating the object under fixed on-axis illumination. In the next step, the captured angular measurements are processed with a filtered backprojection algorithm (FBPJ) [2]. The result of the processing is the reconstruction of a $3 \mathrm{D}$ distribution of the refractive index inside the specimen.

The basic assumption underpinning the FBPJ algorithm is that probing radiation travels through the sample along straight lines. Consequently, the 2D phase maps are interpreted by FBPJ as line integrals of the refractive index evaluated along the illumination directions. As it was proven by many researches, the straight line propagation approximation is accurate for sharp imaging conditions $[3,4]$. However, in the presence of a defocus error, the reconstructed 3D structure suffers from blurring and other diffraction-related deformations. In fact, all conventional microscopy systems use high NA optics to achieve high transverse resolution, which inevitably shrinks the depth of focus (DOF) to a level of a few

*E-mail: j.kostencka@mchtr.pw.edu.pl microns. Thus, defocusing phase images is a key factor affecting the final quality of tomographic reconstructions.

The required sharp imaging condition not only puts restrictions on the spatial extent of an investigated sample but it also implies that the sample rotation has to be done precisely around the axis passing through the centre of the sample. Otherwise, the sample displacement in the direction of the optical axis causes defocusing of the phase maps and thus erroneous tomographic reconstruction. However, precise positioning of the sample in a rotary module of the TPM system is timeconsuming and requires specialized equipment. Even more importantly, in some circumstances accurate alignment of the sample is inaccessible. This is often the case in studies of biological objects [5] and dynamic processes. Moreover, the intentional usage of the off-axis rotation scheme allows minimizing the effect of coherent noise in tomographic reconstruction [6].

This work presents a numerical method for defocus error compensation in TPM, which allows fulfilling the FBPJ requirement for sharp imaging conditions and at the same time reduces the demand for precise aligning of the sample in a TPM system. The proposed method exploits the fact that during an off-axis rotation the sample travels along a circular trajectory around the rotation axis. The vector of the sample displacement can be decomposed into two orthogonal components, one in the direction of the optical axis (axial shift $S_{z}$ ) and the second in the plane parallel to the detector (transverse shift $S_{x}$ ). In the proposed method, the axial displacement, which is related to defocusing, is holographically compensated by numerical propagation of each angular measurement to the in-focus position with a propagation distance $z_{\text {prop }}=-S_{z}(\alpha)$, where $\alpha$ is the angular position of the sample. However, before this can be accomplished, the required amount of the axial displacement has to be determined. In our approach we propose to take advantage of a mutual relation between the displacement vectors: $S_{x}=R \cos \alpha$ and $S_{z}=R \sin \alpha$, where coordinates $(R, \alpha)$ describe the position of the sample in the polar system having its origin at the rotation axis. Thus, $S_{z}$ can be determined from the transverse component $S_{x}$, which in turn can be calculated with very high precision (a fraction 
of a micron) by using one of the tracking techniques, e.g. correlation [7] or the centre-of-mass determination [8] method.

It is worth mentioning that a similar algorithm for defocus error compensation was already presented [6,9]; however, it differs from the approach proposed here in the way of determining axial displacement. In the previous approach $S_{z}$ is detected using a holographic autofocusing technique [10,11], implying a heavy computational load. Moreover, the accuracy of defocus compensation is significantly reduced due to a substantial error of the autofocusing algorithm (accuracy at the level of a few microns) [12]. Furthermore, in contrast to our approach, the autofocusing-based method can be applied solely for objects with pure phase properties.

Before going into details of the proposed defocus compensation method, we briefly explain the measurement process in TPM. Figure 1 presents a conventional TPM measurement system, which is based on Mach-Zehnder configuration [13]. The collimated laser beam is divided by a beam splitter BS into an object and a reference beam. The object beam passes through the sample which is attached to the rotary holder and immersed in an index matching liquid IC. Next, the object wave is imaged by a microscope objective $\mathrm{MO}$ on a CCD detector, where it interferes with the reference beam forming an on-axis hologram. To obtain quantitative phase images a mirror M3 in a reference arm is attached to the piezoelectric transducer (PZT), allowing implementation of the temporal phase shifting method [14].

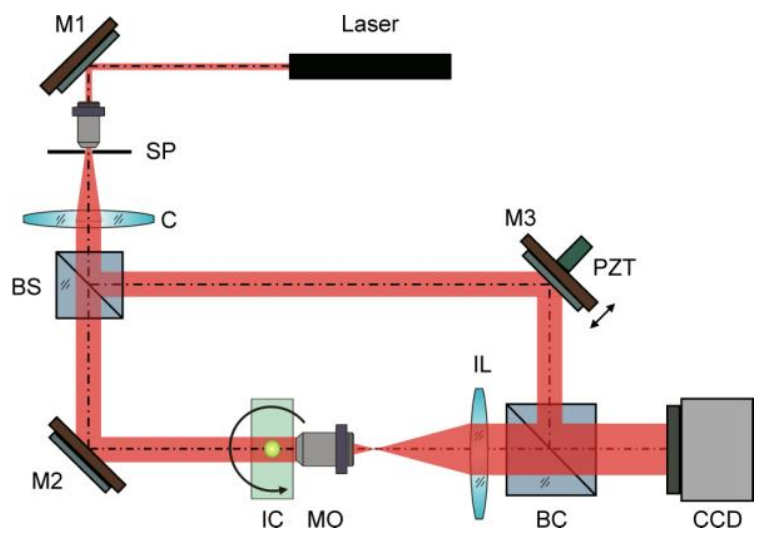

Fig. 1. The scheme of Mach-Zehnder holographic setup.

Using the TPM system a set of phase images is obtained as the object is gradually rotated by $180^{\circ}$. After removing $2 \pi$ ambiguity, phase images are subjected to the FBPJ algorithm, which can be expressed via:

$$
\mathrm{O}(x, y, z)=\int_{0}^{2 \pi+\infty} \int_{-\infty}^{+\infty}\left|f_{x}\right| \tilde{\varphi}_{\alpha}\left(f_{x}, y\right) \exp \left\{\mathrm{i} 2 \pi f_{x} x\right\} d f_{x} d \alpha,
$$

where $\tilde{\varphi}_{\alpha}\left(f_{x}, y\right)$ denotes the 1D Fourier transform of the phase map $\varphi_{\alpha}(x, y)$ calculated in $\mathrm{x}$-direction, and $f_{x}$ is the spatial frequency. The last step of tomographic evaluation is scaling to the refractive index values:

$$
\Delta n(x, y, z)=\frac{\lambda}{2 \pi \Delta_{x}} O(x, y, z)
$$

where $\Delta_{x}$ is the sampling pitch in the object space.

Providing that the phase measurements $\varphi_{\alpha}$ are sharply focused, the FBPJ algorithm delivers an accurate reconstruction of the $3 \mathrm{D}$ sample. However, as it was already mentioned, in the case of the off-axis rotation, which is schematically presented in Fig. 2, the inevitable defocusing causes degradation of the reconstruction.

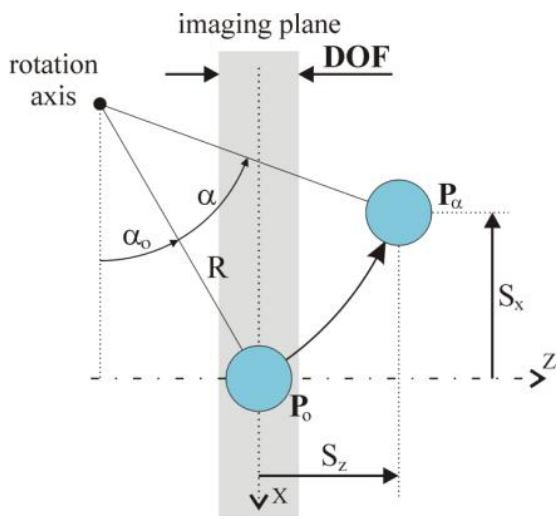

Fig. 2. Schematic diagram of the sample trajectory during rotation.

As it can be noticed from Fig. 2, the amount of defocusing in individual measurements can be deduced from the transverse displacement of the sample: $S_{x}(\alpha)=R\left[\cos \left(\alpha+\alpha_{o}\right)-\cos \alpha_{o}\right]$, where $R$ depicts the radius of the rotation and $\alpha_{o}$ is the initial angular position of the sample. By tracking the sample position in the images and fitting a cosine function to $S_{x}$, we can efficiently compute $S_{z}$ via:

$$
S_{z}(\alpha)=R^{f i t}\left[\sin \left(\alpha+\alpha_{o}^{f i t}\right)-\sin \alpha_{o}^{f i t}\right],
$$

where $R^{\text {fit }}$ and $\alpha_{o}^{\text {fit }}$ are parameters obtained from the fitting. The detection of $S_{x}$ can be handled e.g. with a correlation method [7], which consists in finding the maximum of a cross-correlation peak between two successive angular images. The method provides the accuracy of tracking on the level of one micron [7]. It is notable that the final accuracy of $S_{z}$ estimation is even improved with respect to this value. This is owing to the averaging effect of fitting the cosine to the tracking results obtained for multiple measurements $\varphi_{\alpha}(x, y)$.

The proposed defocus compensation method was experimentally tested with a tomographic measurement of an optical microtip [15] (Fig. 3). For this sample, 90 measurements were taken in a range of $180^{\circ}$ using the TPM system depicted in Fig. 1. 


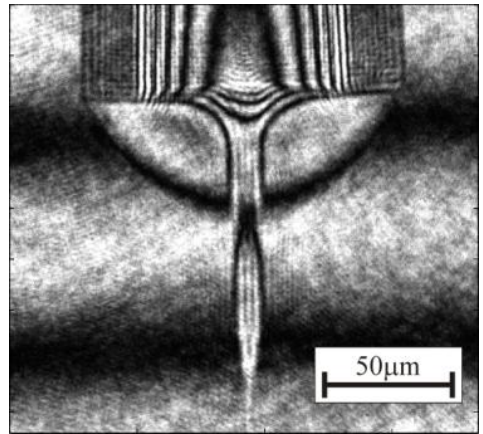

Fig. 3. Exemplary on-axis hologram of a polymer microtip manufactured at the extremity of a fiber.

After unwrapping, the correlation-based transverse tracking method was employed, giving the vector shown in Fig. 4 with a dotted blue line. By calculating the cosine fit to $S_{x}$ (red line) and evaluating Eq. 3, the amount of the axial sample displacement was obtained (the green line in Fig. 4). The estimated $S_{z}$ was used for the compensation of defocus through calculation of an inverse diffraction formula [16] with a propagation distance $z_{\text {prop }}(\alpha)=-S_{z}(\alpha)$. Afterwards, the corrected phase maps were processed with the conventional FBPJ algorithm. The finally obtained 3D reconstructions, calculated with and without defocus compensation, are presented in Figs. 5(a-c) and (d-f), respectively. In Figs. 5(a-c) the reconstruction of the core in the microtip is clearly visible, while in Figs. 5(d-f) several false artefacts have been reconstructed.

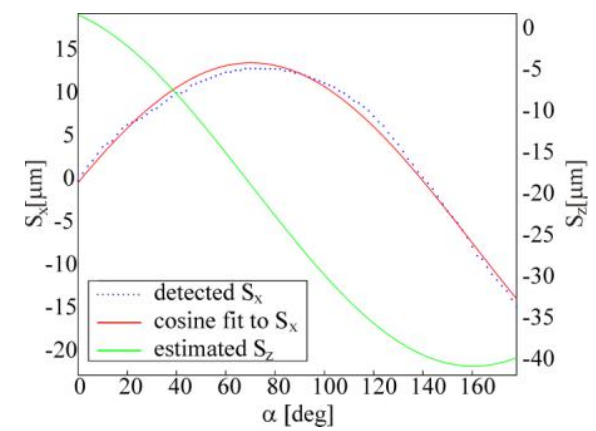

Fig. 4. The charts of axial and transverse displacements.

In conclusion, the proposed defocus compensation method enables high quality reconstruction, even in the presence of relatively strong defocusing of phase images (here maximum defocus $\max \left[\left|S_{z}\right|\right]=40 \mu \mathrm{m}$ ). The proposed method features high accuracy of axial displacement determination and low computational complexity.

This work was founded by the project TEAM/2011-7/7 of the Foundation for Polish Science co-financed from the European Funds of Regional Development and in part by the statutory funds.
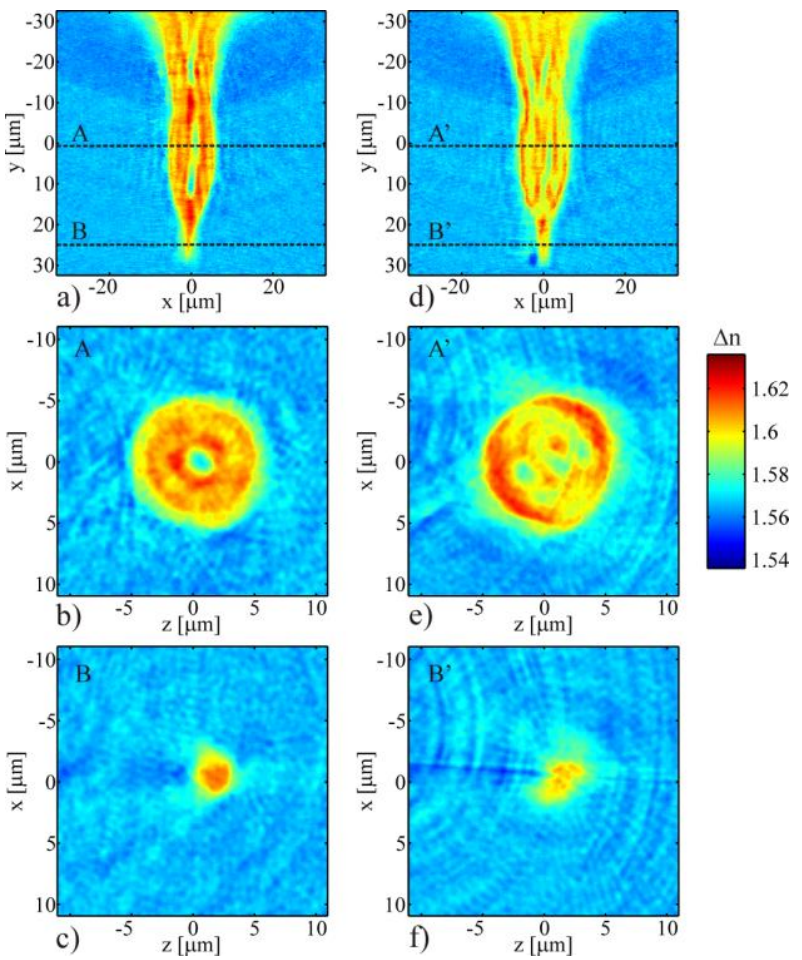

Fig. 5. Vertical and horizontal cross-sections of 3D reconstructions of a microtip obtained with (a-c) and without defocus correction (d-f).

\section{References}

[1] S. Kou, C. Sheppard, Opt. Lett. 33, 2362 (2008), http://dx.doi.org/ 10.1364/OL.33.002362

[2] A. C. Kak and M. Slaney, Principles of Computerized Tomographic Imaging (New York, SIAM 2001)

[3] T.C. Wedberg, J.J. Stamnes, W. Singer, Appl. Opt. 34, 6575 (1995) http://dx.doi.org /10.1364/AO.34.006575

[4] J. Kostencka, T. Kozacki, Proc. SPIE 9132, 91320M (2014), http:// dx.doi.org/10.1117/12.2052227

[5] A. Kuś et al., J. Biomed. Opt. 19, 46009 (2014), http://dx.doi.org/ 10.1117/1.JBO.19.4.046009

[6] J. Kostencka, T. Kozacki, M. Dudek, M. Kujawińska, Opt. Exp. 22, 5731 (2014), http://dx.doi.org/10.1364/OE.22.005731

[7] W. Górski, Opt. Eng. 45, 125002 (2006),http://dx.doi.org/10.1364/ OL.32.001977

[8] F. Charrière et al., Opt. Lett. 31, 178 (2006), http://dx.doi.org/ 10.1364/OL.31.000178

[9] Y. Jeon, C.K. Hong, Opt. Eng. 48, 105801 (2009), http://dx.doi.org/ $10.1117 / 1.3242833$

[10] P. Langehanenberg, B. Kemper, D. Dirksen, G. von Bally, Appl. Opt. 47, D176 (2008), http://dx.doi.org/10.1364/AO.47.00D176

[11] J. Kostencka, T. Kozacki, K. Liżewski, Opt. Commun. 297, 20 (2013), http://dx.doi.org/10.1016/j.optcom.2013.01.078

[12] K. Liżewski, S. Tomczewski, T. Kozacki, J. Kostencka, Appl. Optics 53, 2446 (2014), http://dx.doi.org/10.1364/AO.53.002446

[13] T. Kozacki, M. Józwik, R. Józwicki, Opto-Electron. Rev. 17, 58 (2009), http://dx.doi.org/10.2478/s11772-009-0005-Z

[14] I. Yamaguchi, T. Zhang, Opt. Lett. 22, 1268 (1997),.

[15] M. Kujawińska et al., Proc. SPIE 8494, 849404 (2012), http://dx.doi.org/10.1117/12.940779

[16] T. Kozacki, K. Falaggis, M. Kujawińska, Appl. Opt. 51, 7080 (2012) http://dx.doi.org/10.1364/AO.51.007080 